\title{
Computationally Efficient Changepoint Detection for a Range of Penalties
}

\author{
Kaylea Haynes ${ }^{1 \dagger}$, Idris A. Eckley ${ }^{2}$ and Paul Fearnhead ${ }^{2}$ \\ ${ }^{1}$ STOR-i Centre for Doctoral Training, Lancaster University \\ ${ }^{2}$ Department of Mathematics and Statistics, Lancaster University \\ ${ }^{\dagger}$ Correspondence: k.haynes1@lancaster.ac.uk
}

October 23, 2015

\begin{abstract}
In the multiple changepoint setting, various search methods have been proposed which involve optimising either a constrained or penalised cost function over possible numbers and locations of changepoints using dynamic programming. Recent work in the penalised optimisation setting has focussed on developing an exact pruning-based approach which, under certain conditions, is linear in the number of data points. Such an approach naturally requires the specification of a penalty to avoid under/over-fitting. Work has been undertaken to identify the appropriate penalty choice for data generating processes with known distributional form, but in many applications the model assumed for the data is not correct and these penalty choices are not always appropriate. To this end we present a method that enables us to find the solution path for all choice of penalty values across a continuous range. This permits an evaluation of the various segmentations to identify a suitable penalty choice. The computational complexity of this approach can be linear in the number of data points and linear in the difference between the number of changepoints in the optimal segmentations for the smallest and largest penalty values.
\end{abstract}


Keywords: Penalised Likelihood, Structural Change, Dynamic Programming, Segmentation, PELT

\section{Introduction}

Changepoints are considered to be those points in a data sequence where we observe a change in the statistical properties. For example assume we have data, $y_{1}, \ldots, y_{n}$, that have been ordered based on some covariate information, for example by time or by position along a chromosome. For clarity we will assume we have time-series data in the following. Our timeseries will have $m$ changepoints with locations $\tau_{1: m}=\left(\tau_{1}, \ldots, \tau_{m}\right)$ where each $\tau_{i}$ is an integer between 1 and $n-1$ inclusive. We assume that $\tau_{i}$ is the time of the $i$ th changepoint, so that $\tau_{1}<\tau_{2}<\ldots<\tau_{m}$. We set $\tau_{0}=0$ and $\tau_{m+1}=n$ so that the changepoints split the data into $m+1$ segments with the $i$ th segment containing the data points $y_{\left(\tau_{i-1}+1\right): \tau_{i}}=\left(y_{\tau_{i-1}+1}, \ldots, y_{\tau_{i}}\right)$. There are many different approaches to changepoint detection; see Frick et al. (2014), Jandhyala et al. (2013), Fryzlewicz (2014) and references therein. One common approach is to define a cost for a given segmentation of the data such as the negative log-likelihood (Chen and Gupta, 2000), quadratic loss (Rigaill, 2010) or the minimum descriptive length (Davis et al., 2006). Typically this cost is based on first defining a segment-specific cost function, which we denote as $\mathcal{C}\left(y_{s+1: t}\right)$ for a segment which contains data-points $y_{s+1: t}$. We then sum this segment-specific cost function over the $m+1$ segments. A natural way to then estimate the number and position of the changepoints would be to minimise the resulting cost over all segmentations. Note that, whilst formulated differently, binary segmentation procedures (Scott and Knott, 1974; Olshen et al., 2004) can be viewed as approximately minimising such a cost (see Killick et al., 2012, for more discussion). From herein we use optimal in the sense that the segmentations are solutions of the constrained minimisation problem, i.e., if we have $m$ changepoints then the location of these changepoints are such that they minimise the cost of segmenting the data with $m$ changepoints.

Directly minimising such a cost function will generally result in overfitting, as for many choices of cost function adding a changepoint always reduces the overall cost. There are two potential approaches to avoiding such overfitting. The first of these would be to constrain the optimisation by fixing the maximum number of changepoints that can be found. The 
corresponding constrained minimisation problem is:

$$
Q_{m}\left(y_{1: n}\right)=\min _{\tau_{1: m}}\left\{\sum_{i=1}^{m+1}\left[\mathcal{C}\left(y_{\left(\tau_{i-1}+1\right): \tau_{i}}\right)\right]\right\},
$$

with the best segmentation with $m$ changepoints being the one that attains the minimum. If the number of changepoints is unknown then the number of changes, $m$, is often estimated by solving

$$
\min _{m}\left\{Q_{m}\left(y_{1: n}\right)+f(m)\right\}
$$

where $f(m)$ is a suitably chosen penalty term that increases with $m$.

If $f(m)$ is a linear function, that is $f(m)=(m+1) \beta$ with $\beta>0$, then we can jointly estimate the number and the position of the changepoints by solving a penalised minimisation problem (see for example: Lavielle and Moulines, 2000; Lebarbier, 2005; Jackson et al., 2005; Boysen et al., 2009):

$$
Q\left(y_{1: n}, \beta\right)=\min _{m, \tau_{1: m}}\left\{\sum_{i=1}^{m+1}\left[\mathcal{C}\left(y_{\left(\tau_{i-1}+1\right): \tau_{i}}\right)+\beta\right]\right\}
$$

again with the estimated segmentation being the one that attains the minima. This second approach, of directly minimising (1.3) is computationally faster than solving the constrained penalisation problem for a range of the number of changepoints, and then minimising (1.2); however it requires a choice of penalty constant, $\beta$. Note that some choices of penalty include terms that depend on the segment lengths (e.g. Zhang and Siegmund, 2007; Davis et al., 2006). The resulting penalised minimisation problems can also be formulated in terms of minimising a function of the form (1.3) or (1.2), by including the penalty that depends on the segment length within the segment cost.

Many authors have looked at different choices of penalties. If we let $p$ denote the number of additional parameters introduced by adding a changepoint, then popular examples used frequently in the literature include $\beta=2 p$ (Akaike's Information Criterion; Akaike, 1974); $\beta=p \log n$ (Schwarz's Information Criterion; Schwarz, 1978); and $\beta=2 p \log \log n$ (Hannan and Quinn, 1979). More sophisticated penalty approaches include the modified Bayesian 
Information Criterion (mBIC; Zhang and Siegmund, 2007) which accounts for the length of the segments. Whilst these information criteria all have good theoretical properties, they rely on assumptions about the underlying data generating process which gives rise to the data. Unfortunately, in practice there is potential for the modelling assumptions associated with a particular criterion to be violated. Hocking et al. (2013) show that while the mBIC works well for simulated data sets where the model assumptions of the mBIC hold, it does not work as well for real data sets.

An alternative approach is to calculate optimal segmentations with differing numbers of changepoints, and then use some alternative method to evaluate each segmentation. This idea has been suggested by Hocking et al. (2013) who then use annotated training data to learn what choice of penalty is most appropriate for a given application. For recursive methods, such as variants of binary segmentation (Scott and Knott, 1974; Fryzlewicz, 2014) and the method of Fryzlewicz (2012), we obtain segmentations corresponding to a range of different numbers of changepoints with no, or little, additional cost. However, if the aim is to find segmentations that are optimal, in terms of minimising a given cost function, these recursive methods cannot be used. Calculating the range of optimal segmentations can be done using the Segment Neighbourhood algorithm (Auger and Lawrence, 1989), but this comes at a much higher computational cost.

Our contribution is a new algorithm, CROPS, that can compute all optimal segmentations of the penalised minimisation problem as we vary the penalty over some interval. This is similar in spirit to algorithms for variants of penalised regression where, rather than solving a problem for a single penalty value, one calculates the set of solutions obtained as the penalty value varies (Tibshirani and Taylor, 2011; Fryzlewicz, 2012; Zhou and Lange, 2013).

The CROPS algorithm uses a simple relationship between the solutions of the penalised minimisation problem and those of the constrained minimisation problem to find a set of distinct penalty values such that each solution corresponds to either a different segmentation, or will rule out the possibility of an optimal segmentation under the penalised cost with a certain number of changepoints. We show how the computational cost of the method can be improved by storing and reusing certain values that are calculated when solving the penalised cost problem for the earlier choices of the penalty values. The output of CROPS is similar to that of Segment Neighbourhood, but it can be substantially, even orders of magnitude, 
faster.

The article is organised as follows. In Section 2 we introduce the changepoint model and review various ways of detecting multiple changes using both a constrained and a penalised approach. In Section 3 we propose our method for running the detection algorithms over a range of penalty values, and give a bound on its computational cost. Our method will be demonstrated in simulation studies and real data examples in Section 4 and Section 5.

\section{Background}

\subsection{Segment Costs}

To define the cost of a segmentation we need to specify a segment-specific cost. A common approach, used for example in penalised likelihood (Braun and Muller, 1998) and minimum description length (Davis et al., 2006) methods, is to introduce a model for the data within a segment. This will define a log-likelihood for the data that depends on a segment-specific parameter. The cost can then be chosen proportional to minus the maximum of this $\log$ likelihood, where we maximise out the segment-specific parameter. The form of this cost will then depend on both modelling assumptions about the distribution of the data points, and also the type of change that we are attempting to detect.

To make this idea concrete, consider the following setting, that we will revisit in the simulation and real-data examples. If we model the data within a segment as being independent and identically distributed, drawn from a Gaussian distribution with mean $\mu$ and variance $\sigma^{2}$, then the log-likelihood of data $y_{(s+1): t}$, up to a common additive constant, would be

$$
\ell\left(y_{(s+1): t} ; \mu, \sigma\right)=-\frac{(t-s)}{2} \log \left(\sigma^{2}\right)-\frac{1}{2 \sigma^{2}} \sum_{j=s+1}^{t}\left(y_{j}-\mu\right)^{2}
$$

For detecting a change in mean calculating the segment cost involves using minus twice the log-likelihood after maximising over $\mu$. This gives the segment cost:

$$
\mathcal{C}\left(y_{(s+1): t}\right)=\sum_{j=s+1}^{t}\left(y_{j}\right)^{2}-\frac{\left(\sum_{j=s+1}^{t} y_{j}\right)^{2}}{n} .
$$


Similarly for detecting a change in both mean and variance, calculating the segment cost would involve using minus twice the log-likelihood after maximising over both $\mu$ and $\sigma$. This gives a segment cost,

$$
C\left(y_{(s+1): t}\right)=(t-s)\left\{\log \left[\frac{1}{t-s} \sum_{j=s+1}^{t}\left(y_{j}-\frac{1}{t-s} \sum_{i=s+1}^{t} y_{i}\right)^{2}\right]+1\right\} .
$$

\subsection{Finding optimal segmentations}

We now briefly review existing approaches for finding optimal segmentations within the literature.

\subsubsection{Segment-Neighbourhood}

Auger and Lawrence (1989) introduced the Segment Neightbourhood (SN) search method which is used to solve the constrained problem in (1.1). This method involves specifying the maximum number of changepoints to allow, $M$, and then calculating the cost of all possible optimal segmentations with 0 to $M$ changepoints. The optimal number of changepoints can then be calculated by (1.2). The computational cost for this method is $\mathcal{O}\left(M n^{2}\right)$ and thus this method scales poorly when analysing large data sets with a large number of possible changepoints.

\subsubsection{Optimal Partitioning}

In order to solve the penalised minimisation problem in (1.3), Jackson et al. (2005) introduced a method also based on dynamic programming: Optimal Partitioning (OP). OP is a recursive process which relates the minimum value of (1.3) to the cost of the optimal segmentation of the data prior to the last changepoint plus the cost of the segment from the last changepoint to the current time point. For the data up to time $s, y_{1: s}$, we let $\boldsymbol{\tau}_{\boldsymbol{s}}$ be the set of all possible number and position of changepoints for segmenting the data: $\boldsymbol{\tau}_{\boldsymbol{s}}=\left\{\boldsymbol{\tau}: 0=\tau_{0}<\tau_{1}<\cdots<\right.$ $\left.\tau_{m}<\tau_{m+1}=s\right\}$. If we denote the minimisation of $(1.3)$ for data $y_{1: t}$ by $F(t)=Q\left(y_{1: t} ; \beta\right)$, with $F(0)=0$, then this can be calculated recursively by: 


$$
F(t)=\min _{\tau \in \tau_{t}}\left\{\sum_{i=1}^{m+1}\left[\mathcal{C}\left(y_{\left(\tau_{i-1}\right)+1: \tau_{i}}\right)+\beta\right]\right\}=\min _{s \in\{0, \ldots, t-1\}}\left\{F(s)+\mathcal{C}\left(y_{(s+1): y}\right)+\beta\right\} .
$$

This recursion can be interpreted as stating that the minimum cost of segmenting $y_{1: t}$, given the last changepoint is at time $s$, is the optimal cost for segmenting data up to time $s$ plus the cost of adding a changepoint and the cost for the segment $y_{(s+1): t}$. The value of $s$ which attains the minimum of (2.3) is the position of the last changepoint in the optimal segmentation of $y_{1: t}$. These recursions are solved for $t=1,2, \ldots, n$ with computational cost $\mathcal{O}\left(n^{2}\right)$. Extracting the set of changepoints in the optimal segmentation is achieved by a simple recursion backwards through the data.

\subsection{Pruning Methods}

There has been work in improving these methods through pruning techniques. Killick et al. (2012) introduced a modification of OP; Pruned Exact Linear Time (PELT). This method uses inequality based pruning to remove values of $\tau$ which can never be minima from the minimisation performed at each iteration of the OP algorithm. Killick et al. (2012) show that, under certain regularity conditions, the expected computational cost of PELT is $\mathcal{O}(n)$. Recently Maidstone et al. (2014) proposed an alternative functional based pruning method for OP, FPOP which has an empirical cost of $\mathcal{O}(n)$. This is similar to the pDPA method proposed by (Rigaill, 2010) who use this functional pruning in Segment Neighbourhood search. pDPA has an empirical cost of $\mathcal{O}(n \log n)$. The disadvantage of both pDPA and FPOP is that they only work in situations where we only have changes in one parameter.

\section{Algorithm for a range of penalty values}

In this section we propose a method which solves the penalised optimisation problem (1.3) for a range of penalty values, $\beta$. This method finds the optimal segmentations for a different number of segments without incurring as large a computational cost as solving the constrained optimisation problem for a range of $m$ (the number of changepoints). To achieve this we 
use a relationship between the penalised and constrained optimisation problems in order to sequentially choose values of $\beta$ for which the penalised optimisation needs to be solved. This algorithm can be used within any approach to solving the penalised optimisation problem, which we will define as CPD (as in Change Point Detection) for the remainder of this paper.

\subsection{Link between optimisation problems}

As before, we have $Q_{m}\left(y_{1: n}\right)$ as the minimum cost for the constrained optimisation problem (1.1) and $Q\left(y_{1: n}, \beta\right)$ as the minimum cost of the penalised optimisation problem (1.3). These costs can be linked by defining the minimum cost for the penalised optimisation problem subject to the number of changepoints being $m$ :

$$
P_{m}(\beta)=Q_{m}\left(y_{1: n}\right)+(m+1) \beta
$$

Then we have, for any $\beta$,

$$
Q\left(y_{1: n}, \beta\right)=\min _{m} P_{m}(\beta)
$$

Figure 1 shows example $P_{m}(\beta)$ lines, and the corresponding $Q\left(y_{1: n}, \beta\right)$ curve for a range of penalty values, $\beta \in[5.54,11]$, we discuss interval choice in Section 3.3. There are a few important points of interest to note from this plot. Firstly we can clearly see the relationship between the constrained and penalised problems. For example it is evident that using a penalty, $\beta=10$ and minimising a penalised cost function gives the same optimal segmentation as solving the constrained optimisation problem with $m=7$. Additionally we can see that as $\beta$ increases the optimal number of changepoints decreases. By looking at the dashed lines we can see that not all of the possible number of changes are optimal for some $\beta$. For our example segmentations with $m=9,11,12,14$ or 15 are never optimal choices for any $\beta$.

Additionally in Figure 1 we can see that the penalty values can be partitioned into intervals which all have the same value of $m$. For instance for all $\beta \in[8.38,9.22]$ the resulting $m$ is 8. This suggests that if we can learn the boundaries of these intervals, we can use that information to solve the penalised optimisation problem for values of $\beta$ which will correspond to different optimal segmentations. In particular we only needed to run CPD for the penalty 


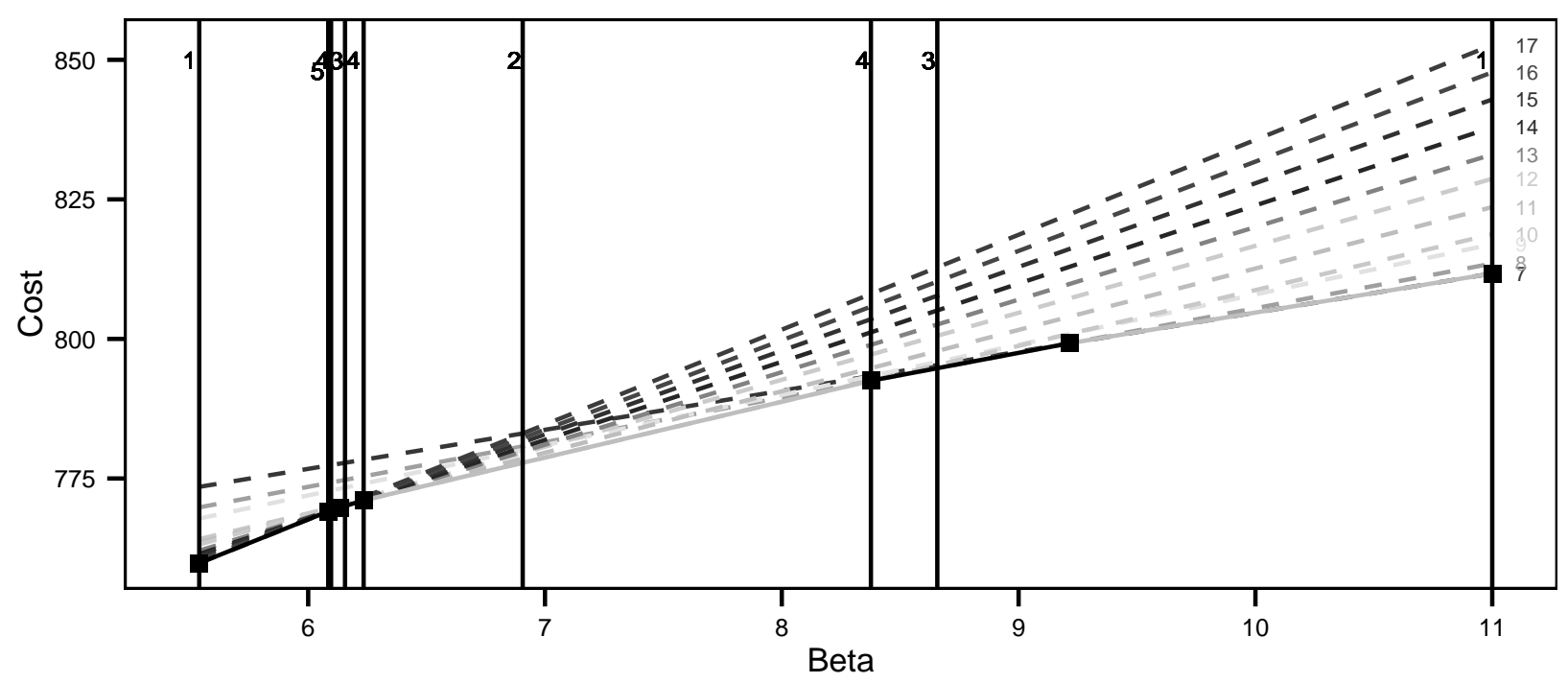

Figure 1: Graphical representation of the relationship between the constrained and penalised approaches. The dashed lines are the costs associated with a different number of changepoints plotted against different penalty terms $\beta$ (3.1). The numbers on the right hand side are the number of changes detected. The solid dark line shows the optimal value of $Q\left(y_{1: n}, \beta\right)$ over the range of $\beta$. The solid line is split in to 6 subregions highlighted by different shades and the black squares. These indicate the intervals where the optimal number of changepoints is the same for all values of the penalty within the interval. The set of $\beta$ values for which CPD was run to find all optimal segmentations for $\beta \in[5.54,11]$ are shown by the vertical lines, interval choice is discussed in Section 3.3. The numbers at the top represent the order in which we use the penalty value, note the same numbers represent penalties run in the same step.

values indicated on the plot by the vertical lines in order to find all optimal segmentations for $\beta \in[5.54,11]$. The next Section describes how we find these values of $\beta$.

\subsection{Theoretical Results}

We now consider the case where we have solved the penalised optimisation problem for two values of penalty, $\beta_{0}$ and $\beta_{1}$.

For any $\beta$ we let $m(\beta)$ be the number of changepoints in the segmentation that is optimal for solving the penalised optimisation problem with penalty $\beta$. If there is more than one optimal segmentation, we let $m(\beta)$ be the smallest number of changepoints in those optimal segmentations. Note that, trivially, $m(\beta)$ will be a non-increasing function.

Theorem 3.1. Let $\beta_{0}<\beta_{1}$. 
(1) If $m\left(\beta_{0}\right)=m\left(\beta_{1}\right)$ then $m(\beta)=m\left(\beta_{0}\right)$ for all $\beta \in\left[\beta_{0}, \beta_{1}\right]$.

(2) If $m\left(\beta_{0}\right)=m\left(\beta_{1}\right)+1$, define

$$
\beta_{\text {int }}=\frac{Q_{m\left(\beta_{1}\right)}\left(y_{1: n}\right)-Q_{m\left(\beta_{0}\right)}\left(y_{1: n}\right)}{m\left(\beta_{0}\right)-m\left(\beta_{1}\right)} .
$$

Then $m(\beta)=m\left(\beta_{0}\right)$ if $\beta \in\left[\beta_{0}, \beta_{\text {int }}\right)$ and $m(\beta)=m\left(\beta_{1}\right)$ if $\beta \in\left[\beta_{\text {int }}, \beta_{1}\right]$.

(3) If $m\left(\beta_{0}\right)>m\left(\beta_{1}\right)+1$, and $m\left(\beta_{\text {int }}\right)=m\left(\beta_{1}\right)$ where $\beta_{\text {int }}$ is defined by (3.3), then $m(\beta)=$ $m\left(\beta_{0}\right)$ if $\beta \in\left[\beta_{0}, \beta_{\text {int }}\right)$ and $m(\beta)=m\left(\beta_{1}\right)$ if $\beta \in\left[\beta_{\text {int }}, \beta_{1}\right]$.

Proof. See the online supplementary material

\subsection{The Changepoints for a Range of PenaltieS (CROPS) algo- rithm}

We now seek to develop a method to find the number of changepoints using different values of the penalty, $\beta$, in a range $\left[\beta_{\min }, \beta_{\max }\right]$. Here we introduce the CROPS algorithm, which sequentially calculates the values of $\beta$.

CROPS begins by first running CPD for penalty values $\beta_{\min }$ and $\beta_{\max }$. Theorem 3.1 then shows that if we have $m\left(\beta_{\min }\right)=m\left(\beta_{\max }\right)$ or $m\left(\beta_{\min }\right)=m\left(\beta_{\max }\right)+1$ we have found all the optimal segmentations for $\beta \in\left[\beta_{\min }, \beta_{\max }\right]$. Otherwise we calculate $\beta_{\text {int }}(3.3)$, the intersection of $P_{m\left(\beta_{\min }\right)}(\beta)$ and $P_{m\left(\beta_{\max }\right)}(\beta)$, then run CPD with this penalty value. By part $(3)$ of Theorem 3.1 we know that if $m\left(\beta_{\text {int }}\right)=m\left(\beta_{\max }\right)$ then we have found all the optimal segmentations for $\beta \in\left[\beta_{\text {min }}, \beta_{\text {max }}\right]$. Otherwise we can now consider the intervals $\left[\beta_{\text {min }}, \beta_{\text {int }}\right]$ and $\left[\beta_{\text {int }}, \beta_{\text {max }}\right]$ separately, and we repeat this procedure on each of those intervals. This continues until there are no new intervals to consider. We are able to use the results above to work out the optimal number of changepoints for all penalty values within the interval $\left[\beta_{\min }, \beta_{\max }\right]$. Pseudo code for this method can be found in Algorithm 1.

Implementing CROPS requires a somewhat arbitrary choice of interval $\left[\beta_{\min }, \beta_{\max }\right]$. However it is clearly easier to find an appropriate value of the penalty if we choose an interval than if we choose just a single value. Furthermore we show in Section 3.5 that if out interval appears inappropriate, we can extend the interval at little additional computational cost. 


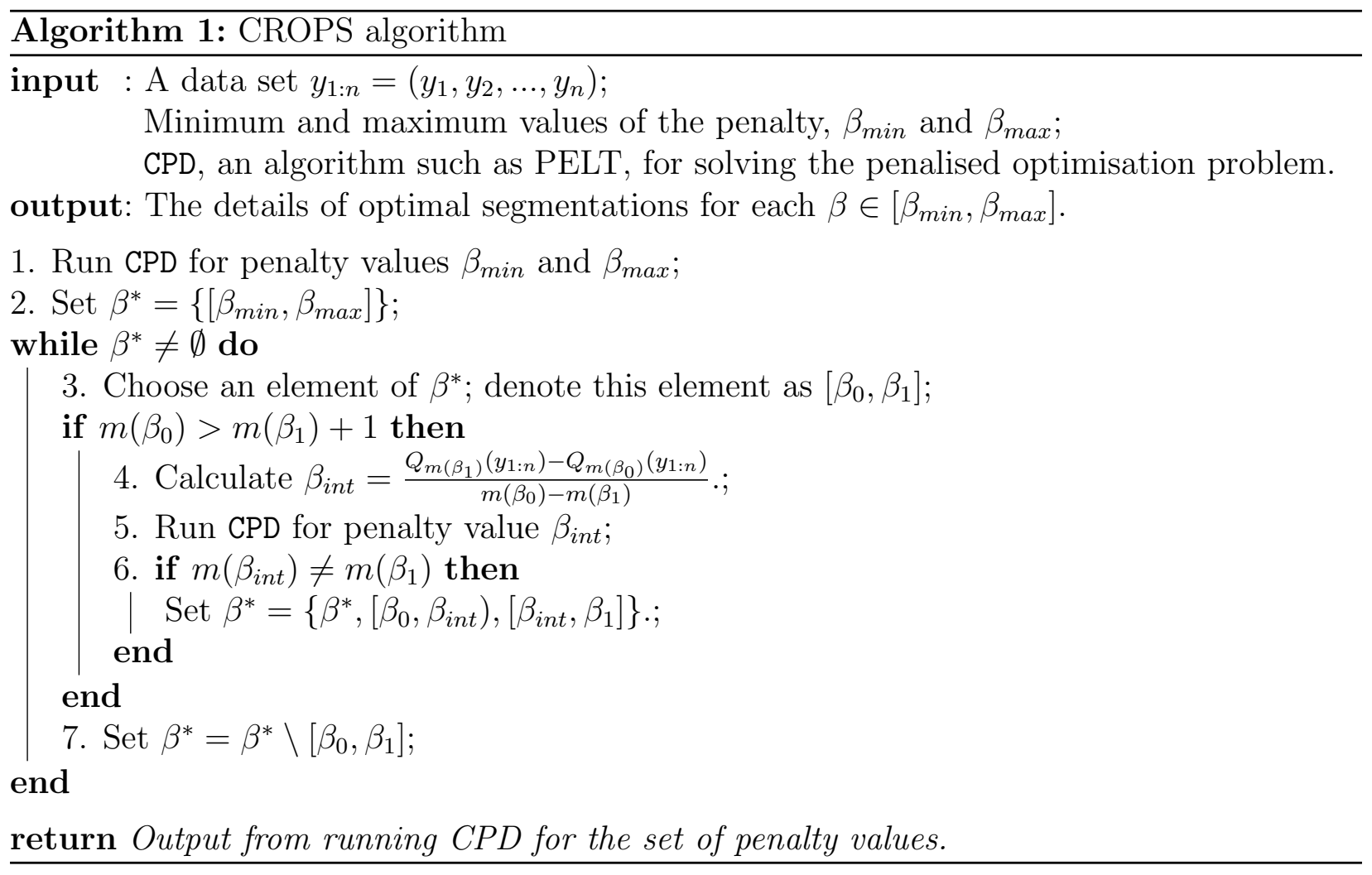

\subsection{The Number of Changepoints that are Optimal for some $\beta$}

For the example in Figure 1 we saw some of the optimal segmentations for specific numbers of changepoints would never be optimal regardless of the penalty value used. Thus using this method will not necessarily get the resulting segmentations for all numbers of changepoints, something which you get when you use segment neighbourhood search.

Lavielle (2005) gives a condition under which a segmentation with $m$ changepoints will be the optimal segmentation for some $\beta$. Assume that segmentations with $m_{1}<\cdots<m_{k}$ changes, for some $k>1$, are optimal as we vary $\beta \in\left[\beta_{\min }, \beta_{\max }\right]$. Let $Q_{i}=Q_{m_{i}}\left(y_{1: n}\right)$, for $i=1, \ldots, k$, be the associated un-penalised cost of these segmentations. We can construct a piece-wise linear line by joining $\left(m_{i}, Q_{i}\right)$ with $\left(m_{i+1}, Q_{i+1}\right)$ for $i=1, \ldots, k-1$. All values of changepoints, $m$, with $m_{1}<m<m_{k}$ and for which there is no optimal segmentation will lie above this line. An example is shown in Figure 2.

One way of expressing this condition is that we will not obtain segmentations for which the average reduction in cost of adding some number of changepoints is more than the average increase in cost of removing some number of changepoints. Consider the example in Figure 


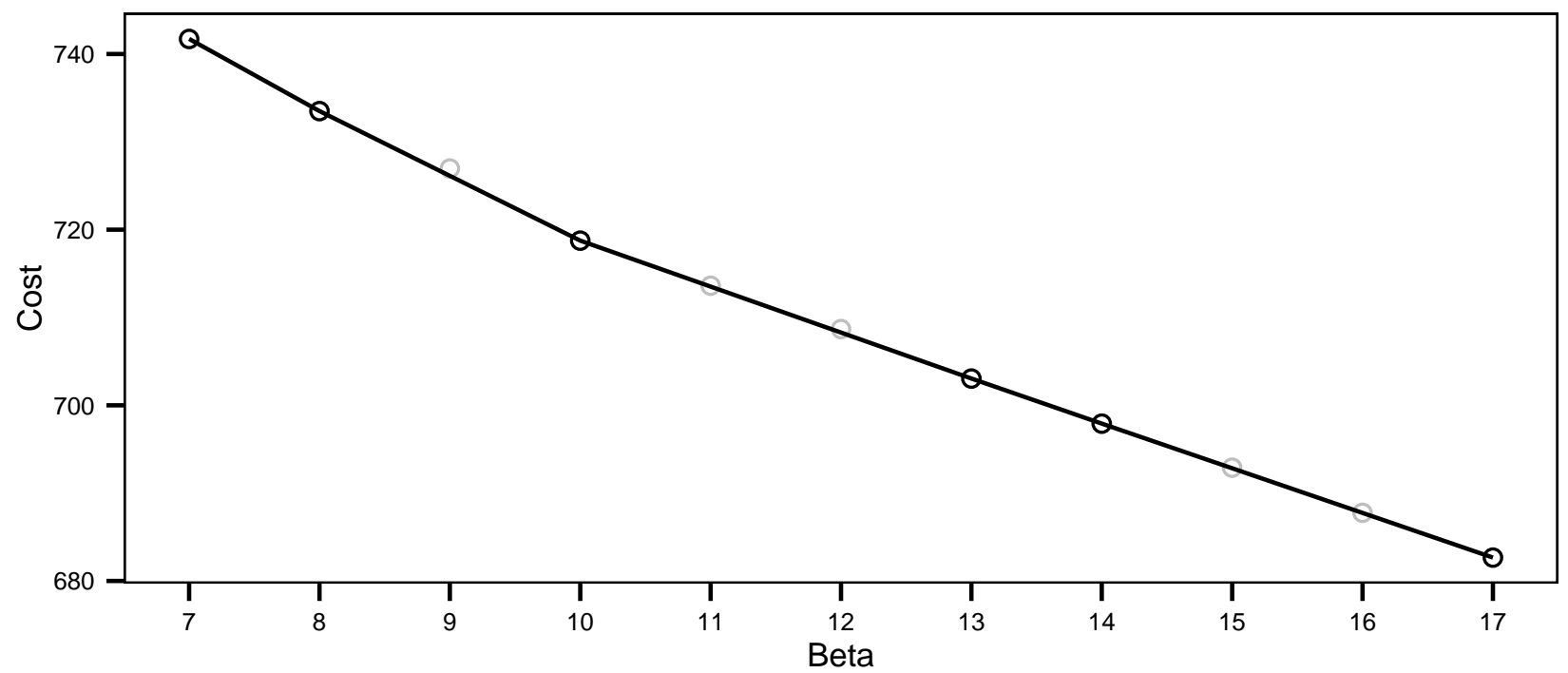

Figure 2: Cost for the segmentations against the number of changepoints. The black circles are the points corresponding to optimal segmentations found by solving the penalised optimisation problem over some range of $\beta$. The grey circles correspond to the segmentations which are not optimal for any penalty.

2. By solving the penalised optimisation problem for a range of $\beta$ we do not find an optimal segmentation with 9 changepoints. This is because the reduction in cost of going from 8 to 9 changepoints is less than for going from 9 to 10 changepoints. It is hard to construct a criteria under which the segmentations not found by solving the penalised optimisation problem would be optimal. In fact Killick et al. (2012) show that any segmentation that is optimal under (1.2) where the penalty function for adding changepoints, $f(m)$, is concave will be the solution to the penalised optimisation problem for some $\beta$.

\subsection{Computational Cost}

We now bound the computational cost of our proposed approach. We do this in terms of the maximum number of times CPD would need to be run. The following theorem shows that this is at most $m\left(\beta_{\min }\right)-m\left(\beta_{\max }\right)+2$ times.

Theorem 3.2. (1) If $m\left(\beta_{0}\right)=m\left(\beta_{1}\right)$ then the maximum number of times that $C P D$ is required to be run to find all the optimal segmentations for $\beta \in\left[\beta_{0}, \beta_{1}\right]$ is $m\left(\beta_{0}\right)$ $m\left(\beta_{1}\right)+2$.

(2) If $m\left(\beta_{0}\right)>m\left(\beta_{1}\right)$ then the number of times that CPD is required to be run to find all 
the optimal segmentations for $\beta \in\left[\beta_{0}, \beta_{1}\right]$ is bounded above by

$$
m\left(\beta_{0}\right)-m\left(\beta_{1}\right)+1
$$

Proof. See online supplementary material.

Often we may choose our interval adaptively. That is we initially choose an interval, $\left[\beta_{\min }^{(1)}, \beta_{\max }^{(1)}\right]$ say. Then, given the set of segmentations we obtain, we may want increase the upper value of interval, reduce the lower value, or both. Assume we wish to increase the upper value of the interval (equivalent reasoning applies for reducing the lower value). Denote the new interval of interest by $\left[\beta_{\min }^{(1)}, \beta_{\max }^{(2)}\right]$ with $\beta_{\max }^{(2)}>\beta_{\max }^{(1)}$. Using the same argument as in the proof of Theorem 3.2, the additional cost will be one run of CPD if $m\left(\beta_{\max }^{(2)}\right)=m\left(\beta_{\max }^{(1)}\right)$, and be at most $m\left(\beta_{\max }^{(1)}\right)-m\left(\beta_{\max }^{(2)}\right)$ runs otherwise. In the latter case, the overall number of runs of CPD is bounded by $m\left(\beta_{\min }^{(1)}\right)-m\left(\beta_{\max }^{(2)}\right)+2$, which is the same bound as if we used the larger interval initially.

\subsubsection{Recycling Calculations}

It is possible to speed up Algorithm 1 by recycling some of the calculations for example if in the situation where we use PELT. As we describe in the online supplementary material, for the PELT algorithm we calculate and store the minimum penalised cost, the number of changepoints in this segmentation for $t=1, \ldots, n$ and the position of the most recent changepoint up to time $t$. If PELT was run with penalty value $\beta$ we denote these values as $F(t, \beta), m(t, \beta)$ and $c p(t, \beta)$ respectively. We can re-use these values from previous runs of PELT to precalculate many of the values for a new run.

Assume we have run PELT with penalty values $\beta_{0}$ and $\beta_{1}$, and are now wanting to run PELT for $\beta_{\text {int }}$ where $\beta_{0}<\beta_{\text {int }}<\beta_{1}$. Before running PELT for the new value we iterate for $t=1, \ldots, n:$

1. If $m\left(t, \beta_{0}\right)=m\left(t, \beta_{1}\right)$ then set $m\left(t, \beta_{\text {int }}\right)=m\left(t, \beta_{0}\right), c p\left(t, \beta_{\text {int }}\right)=c p\left(t, \beta_{0}\right)$ and $F\left(t, \beta_{\text {int }}\right)=$ $F\left(t, \beta_{0}\right)+m\left(t, \beta_{\text {int }}\right)\left(\beta_{\text {int }}-\beta_{0}\right)$.

2. If $m\left(t, \beta_{0}\right)=m\left(t, \beta_{1}\right)+1$ then calculate $a=F\left(t, \beta_{0}\right)+m\left(t, \beta_{0}\right)\left(\beta_{\text {int }}-\beta_{0}\right)$ and $b=$ 


$$
\begin{aligned}
& F\left(t, \beta_{1}\right)+m\left(t, \beta_{1}\right)\left(\beta_{\text {int }}-\beta_{1}\right) \text {. If } a<b \text { then } m\left(t, \beta_{\text {int }}\right)=m\left(t, \beta_{0}\right), c p\left(t, \beta_{\text {int }}\right)=c p\left(t, \beta_{0}\right) \\
& \text { and } F\left(t, \beta_{\text {int }}\right)=a \text {; else } m\left(t, \beta_{\text {int }}\right)=m\left(t, \beta_{1}\right), c p\left(t, \beta_{\text {int }}\right)=c p\left(t, \beta_{1}\right) \text { and } F(t, \beta)=b .
\end{aligned}
$$

We then just need to run PELT to calculate the values of $F\left(t, \beta_{\text {int }}\right), m\left(t, \beta_{\text {int }}\right)$ and $c p\left(t, \beta_{\text {int }}\right)$ for times $t$ that we have not been able to precalculate them.

\section{Simulation Study}

This section shows the performance of CROPS in comparison to other methods which find a range of segmentations. In particular we look at two models: the first being a uniform variance model with a change in mean and the second being a model with both changes in mean and variance. For the change in mean model the quickest method for solving the penalised optimisation problem is FPOP (Maidstone et al., 2014, available from https://rforge.r-project.org/projects/opfp/) and the quickest method for solving the constrained optimisation problem is pDPA (Rigaill, 2010, available in the Segmentor3IsBack R package, Cleynen et al. (2014)). Thus we compare the CROPS using FPOP with pDPA. As described in Section 2.3 neither pDPA or FPOP can be applied when there is more than one parameters for each segment. So for the change in mean and variance we compare CROPS with PELT against Segment Neighbourhood. In this latter case we also compare the speed of CROPS with and without the recycling of calculations introduced in Section 3.5.1.

Since all of these methods optimise exactly, a solution with $m$ changepoints will have the same $m$ changepoints for all of the methods, we only compare the different methods in terms of speed. We are also able to use CROPS to efficiently study and compare some different proposals for the choice of the penalty. Whilst some of these work well when we use the correct model for the data, we show that they can give misleading results when the model is mis-specified, something that is likely to be a feature of real-life applications of changepoint detection.

\subsection{Change in mean}

We simulate data of varying lengths with changepoints distributed uniformly in time but with the constraint that there are at least 20 observations between changepoints. For a 
given value of $n$ we simulate data sets with a fixed number of changepoints, $m=2$. (See the online supplementary material for the cases where we have a linear, $m=n / 100$, and sublinear, $m=\sqrt{n} / 4$, number of changepoints). We generate the segment means from a Normal distribution with mean 0 and standard deviation 2.5 and we let the segment standard deviation be 1. For this model we use the cost function in (2.1).

In the CROPS algorithm we set $\beta_{\min }=4$ and $\beta_{\max }=40$ as indicative values only. For pPDA we set the maximum value of changepoints to be the number of changepoints detected using the smallest value of the penalty value in FPOP. The results are shown in Figure 3.

(a)

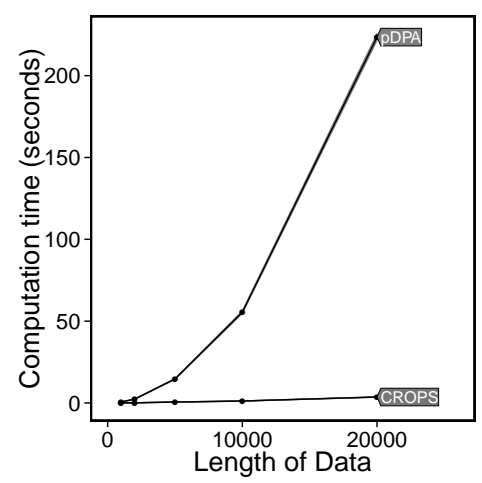

(b)

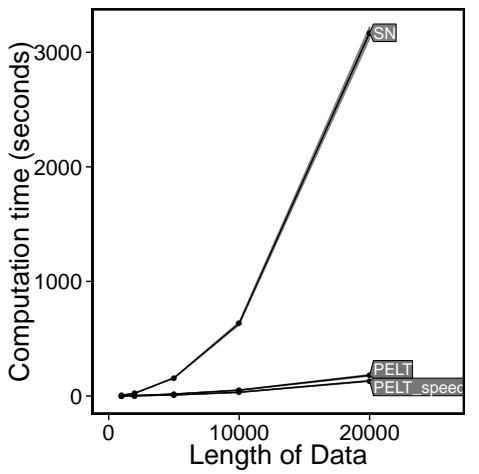

(c)

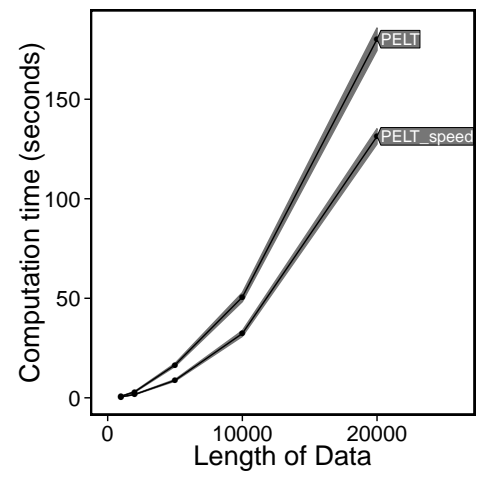

Figure 3: (a) CPU cost for using either pDPA or CROPS with FPOP. (b) CPU cost for using SN, CROPS with PELT and CROPS with PELT with the speed improvements. (c) A close up of PELT and PELT with the speed improvements.

It is evident from Figure 3a that using CROPS with FPOP is substantially quicker than using pPDPA. As the length of the data set increases the gains in speed increase.

\subsection{Change in mean and variance}

To look at models with a change in mean and variance we simulate data as above but this time we generate the segment means from a Normal distribution with mean 0 and standard deviation 2.5, and the segment standard deviations from a Log-Normal distribution with mean 0 and standard deviation $\frac{\log (10)}{2}$. In the case with a fixed number of changepoints we use $m=10$. For this model we use the cost function in (2.2). In the CROPS algorithm we set $\beta_{\min }=14$ and $\beta_{\max }=40$. For $\mathrm{SN}$ we set the maximum value of changepoints to be the number of changepoints detected using the smallest value of the penalty value in FPOP. 
The results can be seen in Figure 3b. Similar to the above results it is evident that CROPS with PELT is much faster than SN. It can be seen in Figure 3c that the addition of the recycling of the calculations (PELT_speed) leads to modest gains in speed.

\subsection{Evaluating the Choice of Penalty}

In this section, we use the change in mean and variance model as above and a mis-specified model. For the mis-specified model, for a segment $k$ we simulate segment standard deviations, $\sigma_{k}^{2}$, and an initial mean value, $\mu_{k}$. If $Y_{t}$ is in segment $k$ then we simulate our data from $Y_{t} \sim$ $N\left(\nu_{t}, \sigma_{k}^{2}\right)$, where $\nu_{t}=\mu_{k}$ if $t$ is the first point in a segment and $\nu_{t+1}=\nu_{t}+\epsilon_{t}, \epsilon_{t} \sim N(0,0.1)$ otherwise. We generate the initial segment means from a Normal distribution with mean 0 and standard deviation 2.5 the segment standard deviations from a Log-Normal distribution with mean 0 and standard deviation $\frac{\log (10)}{2}$. The results for the real and misspecified model are shown in Figures 4 and 5 respectively. To evaluate the penalty choice we initially find the range of $\beta$ values which estimate the correct number of changepoints. For a given simulation scenario $(n=10,000)$ we calculate the average of this range over 100 simulated data sets, and compare this average with the different penalty choices (Figures 4a and 5a). We then look at the proportion of true positive changepoints, $\xi(\mathbf{C} \| \hat{\mathbf{C}})$, and false positive changepoints, $\xi(\hat{\mathbf{C}} \| \mathbf{C})$ (Figures $4 \mathrm{~b}$ and $5 \mathrm{~b})$. To calculate these we define an actual changepoint as detected if we infer a changepoint within 10 time points of its location. Let $\mathbf{C}$ be the vector of $n_{\mathbf{C}}$ true changepoint positions, and $\hat{\mathbf{C}}$ be the vector of $n_{\hat{\mathbf{C}}}$ estimated changepoint positions. If $I(\cdot)$ is an indicator function then

$$
\xi(\mathbf{C} \| \hat{\mathbf{C}})=\frac{\sum_{i=1}^{n_{\mathbf{C}}} I\left(\min _{j}\left\{\left|C_{i}-\hat{C}_{j}\right|\right\} \leq 10\right)}{n_{\mathbf{C}}} \text { and } \xi(\hat{\mathbf{C}} \| \mathbf{C})=1-\frac{n_{\mathbf{C}} \xi(\mathbf{C} \| \hat{\mathbf{C}})}{n_{\hat{\mathbf{C}}}}
$$

For the real model case we can also look at the mean square error (MSE) to evaluate the accuracy of estimates of the segment parameters. That is if $\hat{\theta}_{i}$ is an estimated parameter of the observation at time $i$, and $\theta_{i}$ the true parameter then MSE is $\sum_{i=1}^{n}\left(\hat{\theta}_{i}-\theta_{i}\right)^{2} / n$. We look at MSE for the mean and standard deviation separately (Figure 4c).

From the real model results it can be seen that, in this example, when we have 10 changepoints in the data the optimal value of the penalty lies in a wide interval which increases with data 
size. In this case we can see that the AIC, SIC and Hannan-Quinn penalty values will all over-fit the data. From further simulations (see the online supplementary material) we found that when the number of changepoints increases with the amount of data, the interval in which the optimal penalty value lies decreases as the length of the data increases. In this case the SIC underestimates the number of changes whereas the AIC and Hannan-Quinn penalty term both overestimate the number of changes. When there is a sublinear number of changepoints the optimal penalty value lies in a smaller interval than it did when there was a fixed number of changes. In this case the SIC, AIC and Hannan-Quinn penalty all overestimate the number of changepoints.

In terms of accuracy it is clear to see that both the AIC and Hannan-Quinn penalty detect a lot of false positive changepoints. The SIC penalty outperforms the Hannan-Quinn penalty for estimating the segment parameters. In all cases the MSE for the AIC penalty term was much larger than the other two penalties and thus not shown.

(a) Penalty values

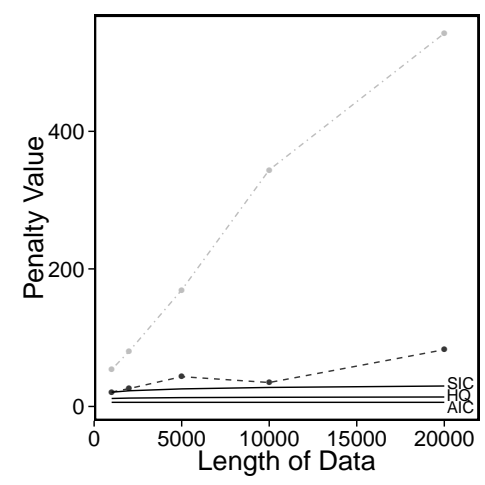

(b) ROC curve

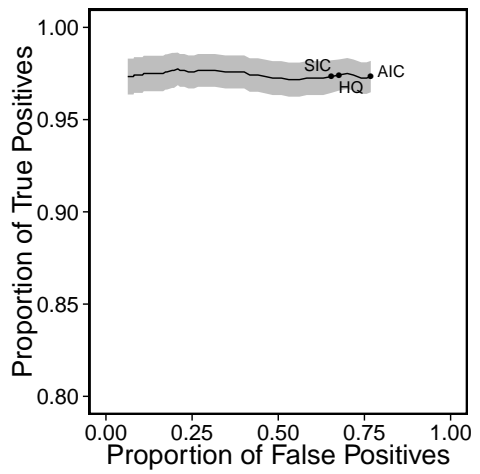

(c) MSE

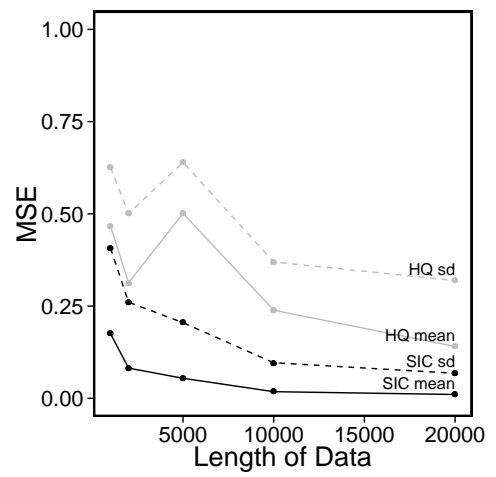

Figure 4: Results for the true model. (a) Average minimum (black, dashed) and maximum (grey, dot-dashed) optimal penalty values in comparison to popular penalty terms in the literature. Solid lines from top to bottom are the SIC, Hannan-Quinn and AIC penalty values. (b) Proportion of true positives against the proportion of false positives for $n=10,000$. (c) MSE for the mean (solid) and the standard deviation (dashed).

We now look at the case where we have the mis-specified model. These results can be seen in Figure 5. It is obvious from these results that the optimal penalty value, in terms of correctly estimating the number of changepoints, is much greater than that for the correctly specified model. It is also much larger than any of SIC, AIC and Hannan-Quinn. From the accuracy 
(a) Penalty values

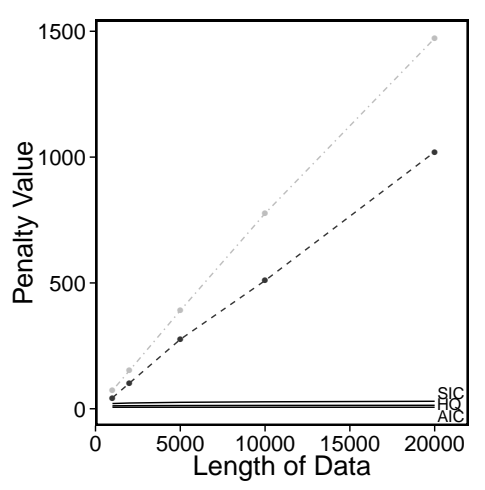

(b) ROC curve

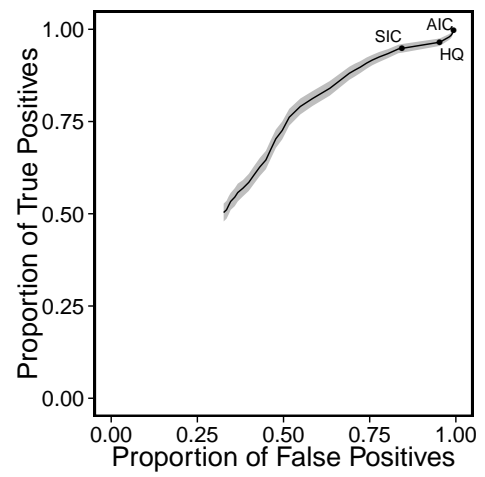

Figure 5: Results for the misspecified model scenario. (a) Average minimum (black, dashed) and maximum (grey, dot-dashed) optimal penalty values in comparison to popular penalty terms in the literature. (b) Proportion of true positives against the proportion of false positives for $n=10,000$.

plot we can see that none of the penalty terms perform well, with them all detecting a large number of false positives.

\section{Application to $\mathrm{Hi}-\mathrm{C}$ data}

Lévy-Leduc et al. (2014) look at detecting genomic regions that interact through the folding and 3-D structure of the chromosome. They achieve this through changepoint detection from a deep sequencing approach called Hi-C. A chromosome is split into a series of windows of consecutive base-pairs on a chromosome. The Hi-C data consists of measurements, $y_{i j}$, of the amount of interaction between window $i$ and window $j$. We expect regions that interact to be contiguous along a chromosome, so the windows are ordered based on position along the chromosome. Then Lévy-Leduc et al. (2014) segment the data into $m+1$ contiguous regions, where $\mathrm{E}\left(Y_{i j}\right)=\mu_{s}$ if gene $i$ and $j$ are both in segment $s$ for some $s=1, \ldots, m+1$; and $\mathrm{E}\left(Y_{i j}\right)=\mu_{0} \approx 0$ if genes $i$ and $j$ are in different segments. Example data from the first 200 windows on Chromosome 16 is shown in Figure 6(a). Note that there is a single measurement for each pair of windows, so we have set $y_{i j}=y_{j i}$. A segmentation produces square regions on the diagonal of the data matrix, corresponding to the measurements between pairs of genes that have been grouped together. 
Lévy-Leduc et al. (2014) formulate the segmentation problem in the form of minimising the penalised cost $\sum_{i=1}^{m+1} \mathcal{C}\left(y_{\tau_{i-1}+1: \tau_{i}}\right)+\beta m$. They consider a range of different segment costs. We will focus on one, where $\mathcal{C}\left(y_{s: t}\right)$ is defined in terms of data $y_{i j}$ with $s \leq i \leq t$ and $j<i$, by

$$
\mathcal{C}\left(y_{s: t}\right)=\min _{\mu}\left[\sum_{i=s+1}^{t} \sum_{j=s}^{i-1}\left(y_{i j}-\mu\right)^{2}\right]+\min _{\mu_{0}}\left[\sum_{i=s}^{t} \sum_{j=1}^{s-1}\left(y_{i j}-\hat{\mu}_{0}\right)^{2}\right] .
$$

This is a non-standard segmentation problem. Brault et al. (2015) show that, under a form of in-fill asymptotics, you can consistently estimate the number of changepoints using $\beta=0$, and this is the choice used in Lévy-Leduc et al. (2014). We will consider using CROPS to study segmentations for a range of penalty values. Note that for this application, the cost function does not satisfy the condition explained in Killick et al. (2012) for PELT, since adding a change doesn't necessarily reduce the cost and thus we use Optimal Partitioning.

Figure 6 shows the results for analysing data from Chromosome 16 (in total over 2.4 million data points corresponding to 2,221 windows). We ran CROPS with the interval $[0,1000]$ which required us to solve the Optimal Partitioning recursions just 34 times, whereas Segment Neighbourhood would have required us to solve an almost identical set of recursions 217 times; thus CROPS reduces the computational cost of the dynamic programming recursions by an order of magnitude.

In order to then pick the best segmentation we use a method suggested by Lavielle (2005), which looks at how the minimum value of the cost changes as we add more changepoints. To do this we plot the un-penalised cost against the number of segments, $m$ (Figure 6b). Initially as we increase $m$ we are likely to be detecting true changes, these will eventually become false positives, and we would expect that detecting a false positive will not lower the cost as much. Thus Lavielle (2005) suggests choosing the point where the decrease in cost due to detecting a further changepoint noticeably changes. This can be thought of as looking for an "elbow" in the plot. In practice such an approach may suggest a plausible range of values for $m$ and these could then be considered in turn as alternative segmentations. Using this approach suggests a segmentation with 200 changepoints. By comparison using $\beta=0$, as suggested by Lévy-Leduc et al. (2014) finds 217 changepoints, and using the SIC penalty produces a segmentation with 214 changepoints. In Figures $6 \mathrm{c}$ and $6 \mathrm{~d}$ we plot two 
(a) 1:200

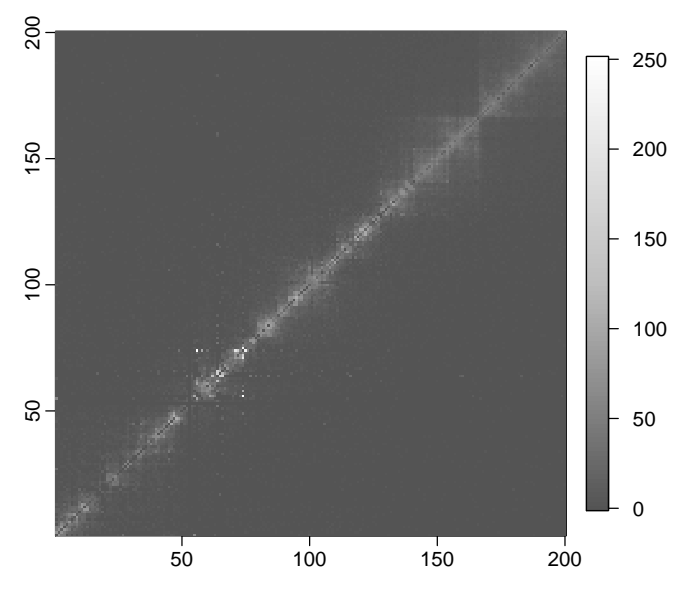

(c) $330: 430$

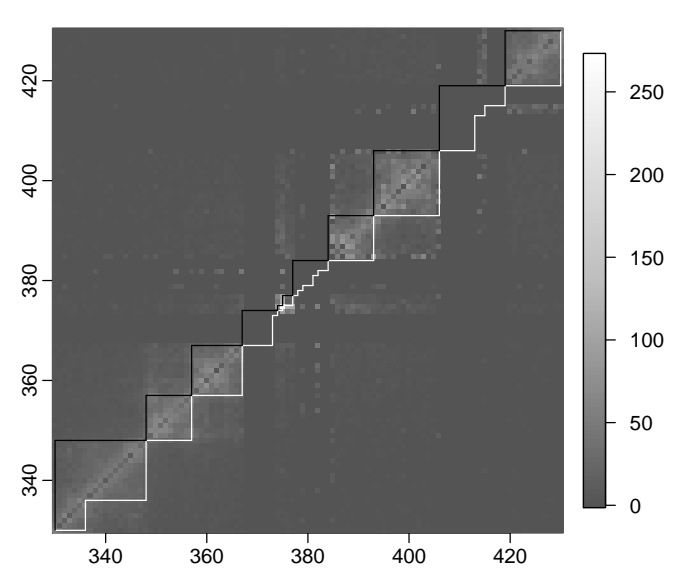

(b) Elbow

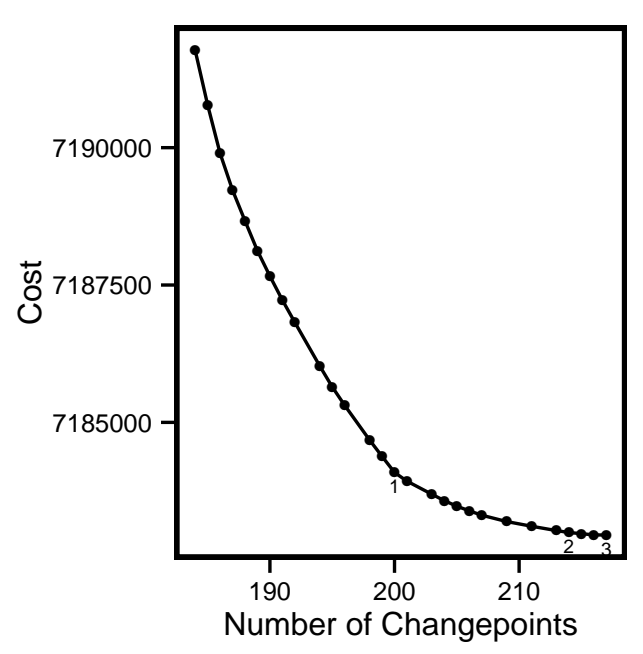

(d) $520: 580$

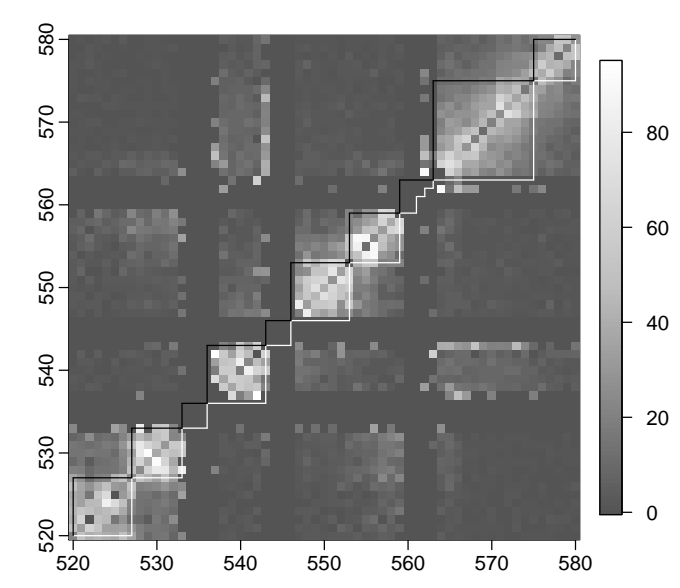

Figure 6: (a) First $200 \times 200$ data points of Chromosome 16. (b) The costs vs number of changepoints for Chromosome 16 where 1 is the point we use as being on the "elbow" and thus refer this to the optimal penalty value, 2 is when we use the SIC penalty and 3 is when the penalty is equal to 0. (c) Close up of the segmentations for windows 330 to 430 . (d) close up of the segmentations for windows 520 to 580. In both cases the black line is our segmentation and the grey line is the segmentation using $\beta=0$. For (c) using SIC gives the same segmentation as $\beta=0$; whilst for (d) using SIC gives the same segmentation as ours. 
regions where there was greatest disparity, between the three segmentations. Plots of other regions with differences in segmentations are in the online supplementary material. The segmentations with the SIC penalty or with $\beta=0$ seem to over-fit the data, introducing changepoints into regions, such as around window 380 or window 560, where there is little signal.

\section{Discussion}

In this paper we have developed a method, CROPS, to obtain the optimal segmentations of data, based on minimising a penalised cost function, for a range of penalty values. For many applications, we believe this is a more appropriate approach to segmenting data than just using a single choice of penalty, such as SIC. In particular, whilst default choices can work well if we have an accurate model for the data within each segment, we have shown that they lack robustness, and can produce poor segmentations, in the presence of model misspecification. We have observed such issues in both a simulation study, and when analysing the genome data.

Minimising the penalised cost function for a range of penalty values is one way of producing a number of different ways of segmenting data, each with a different number of segments. As such, this approach is an alternative to the Segment Neighbourhood search method (and the corresponding pruned method, pDPA), which outputs the optimal segmentation as the number of segments is varied across a suitably chosen range. The advantage of the new approach is one of computational speed, which benefits from the fact that minimising the penalised cost function is a simpler problem to solve than minimising the cost function under a constraint on the number of changepoints, the problem that Segment Neighbourhood solves. In our simulations, CROPS was up to two orders of magnitude quicker than Segment Neighbourhood. One advantage of Segment Neighbourhood is that it produces an optimal segmentation for all numbers of segments in the chosen range, whereas some of these may not be optimal under the penalised cost function for any penalty value, and hence not found via our new method. However the segmentations we do not recover correspond to, for example, ones where adding an extra changepoint leads to a larger change in cost than removing a changepoint. It is hard to construct a sensible criteria under which such segmentations would 
be optimal.

Code implementing CROPS is available in the $\mathrm{R}$ changepoint package, (Killick et al., 2014).

Acknowledgements Haynes gratefully acknowledges funding from EPSRC via the STOR-i Centre for Doctoral Training, and the Defence Science and Technology Laboratory. This research was also supported by the Research Councils UK Energy Programme grant number EP/I016368/1. We thank Vincent Brault for providing access to the Hi-C data used in this article.

\section{References}

Akaike, H. (1974). A New Look at the Statistical Model Identification. IEEE Transactions on Automatic Control, 19(6):716-723.

Auger, I. and Lawrence, C. (1989). Algorithms for the Optimal Identification of Segment Neighborhoods. Bulletin of Mathematical Biology, 51(1):39-54.

Boysen, L., Kempe, A., Liebscher, V., Munk, A., and Wittich, O. (2009). Consistencies and rates of convergence of jump-penalized least squares estimators. Ann. Statist., 37(1):157183.

Brault, V., Delattre, M., Lebarbier, E., Mary-Huard, T., and Lévy-Leduc, C. (2015). Estimating the number of change-points in a two-dimensional segmentation model without penalization. ArXiv e-prints.

Braun, J. V. and Muller, H. G. (1998). Statistical methods for DNA sequence segmentation. Statistical Science, 13:142-162.

Chen, J. and Gupta, A. K. (2000). Parametric statistical change point analysis. Birkhauser.

Cleynen, A., Rigaill, G., and Koskas, M. (2014). Segmentor3IsBack: A Fast Segmentation Algorithm. R package version 1.8 .

Davis, R. A., Lee, T. C. M., and Rodriguez-Yam, G. A. (2006). Structural Break Estimation for Nonstationary Time Series Models. Journal of the American Statistical Association, 101(473):223-239. 
Frick, K., Munk, A., and Sieling, H. (2014). Multiscale change point inference. Journal of the Royal Statistical Society: Series B (Statistical Methodology), 76(3):495-580.

Fryzlewicz, P. (2012). Timethreshold maps: Using information from wavelet reconstructions with all threshold values simultaneously. Journal of the Korean Statistical Society, $41(2): 145-159$.

Fryzlewicz, P. (2014). Wild binary segmenation for multiple change-point detection. Ann. Statist., 42:2243-2281.

Hannan, E. and Quinn, B. (1979). The determination of the order of an autoregression. Journal of the Royal Statistical Society: Series B, 41(2):190-195.

Hocking, T., Rigaill, G., Vert, J.-P., and Bach, F. (2013). Learning sparse penalties for change-point detection using max margin interval regression. In ICML (3), volume 28 of JMLR Proceedings, pages 172-180.

Jackson, B., Scargle, J. D., Barnes, D., Arabhi, S., Alt, A., Gioumousis, P., Gwin, E., Sangtrakulcharoen, P., Tan, L., and Tsai, T. T. (2005). An Algorithm for Optimal Partitioning of Data on an Interval. Signal Processing Letters, IEEE, 12(2):105-108.

Jandhyala, V., Fotopoulos, S., MacNeill, I., and Liu, P. (2013). Inference for single and multiple change-points in time series. Journal of Time Series Analysis.

Killick, R., Eckley, I. A., and Haynes, K. (2014). changepoint: An R package for changepoint analysis. $\mathrm{R}$ package version 2.1.1.

Killick, R., Fearnhead, P., and Eckley, I. A. (2012). Optimal detection of changepoints with a linear computational cost. Journal of the American Statistical Association, 107(500):15901598.

Lavielle, M. (2005). Using penalized contrasts for the change-point problem. Signal Processing, 85(8):1501-1510.

Lavielle, M. and Moulines, E. (2000). Least-squares estimation of an unknown number of shifts in a time series. Journal of Time Series Analysis, 21(1):33-59. 
Lebarbier, E. (2005). Detecting multiple change-points in the mean of gaussian process by model selection. Signal Processing, 85:717-736.

Lévy-Leduc, C., Delattre, M., Mary-Huard, T., and Robin, S. (2014). Two-dimensional segmentation for analyzing hi-c data. Bioinformatics, 30(17):386-392.

Maidstone, R., Hocking, T., Rigaill, G., and Fearnhead, P. (2014). On Optimal Multiple Changepoint Algorithms for Large Data. ArXiv e-prints.

Olshen, A. B., Venkatraman, E. S., Lucito, R., and Wigler, M. (2004). Circular binary segmentation for the analysis of array-based dna copy number data. Biostatistics, 5(4):55772.

Rigaill, G. (2010). Pruned dynamic programming for optimal multiple change-point detection. arXiv preprint arXiv:1004.088\%.

Schwarz, G. (1978). Estimating the Dimension of a Model. Ann. Statist., 6(2):461-464.

Scott, A. J. and Knott, M. (1974). A Cluster Analysis Method for Grouping Means in the Analysis of Variance. Biometrics, 30(3):507-512.

Tibshirani, R. J. and Taylor, J. (2011). The solution path of the generalized lasso. The Annals of Statistics, 39(3).

Zhang, N. and Siegmund, D. O. (2007). A Modified Bayes Information Criterion with Applications to the Analysis of Comparative Genomic Hybridization Data. Biometrics, 63(1):2232.

Zhou, H. and Lange, K. (2013). A path algorithm for constrained estimation. Journal of Computational and Graphical Statistics, 22(2):261-283. 


\section{SUPPLEMENTARY MATERIAL}

\section{Pseudo-code for PELT}

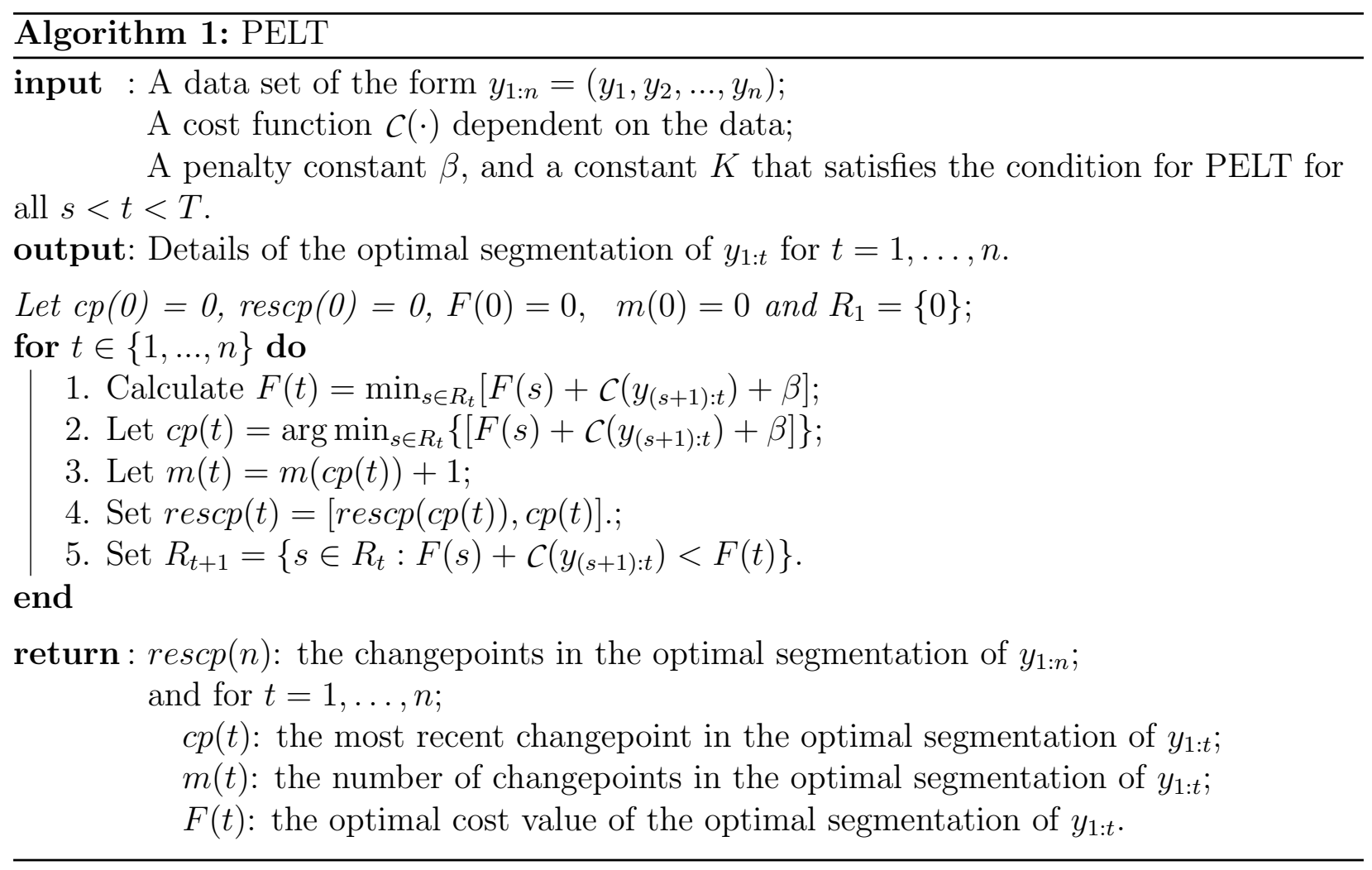

Proof of Theorem 3.1 Proof. To simplify notation, write $m_{0}=m\left(\beta_{0}\right)$ and $m_{1}=m\left(\beta_{1}\right)$.

Part (1) follows immediately from the fact that $m(\beta)$ is a decreasing function.

For part (2), note that as $m(\beta)$ is decreasing, then $m(\beta)$ will be equal to either $m_{0}$ or $m_{1}$ for all $\beta \in\left[\beta_{0}, \beta_{1}\right]$. Using (3.1), to find the interval of values for which $m(\beta)=m_{0}$ we need to find the values of $\beta$ for which $P_{m_{0}}(\beta)<P_{m_{1}}(\beta)$. The value $\beta_{\text {int }}$ is just the solution to $P_{m_{0}}(\beta)=P_{m_{1}}(\beta)$. This gives the required result.

For part (3), first note that as $m(\beta)$ is decreasing, then as $m\left(\beta_{\text {int }}\right)=m_{1}$ we must have $m(\beta)=m_{1}$ for all $\beta \in\left[\beta_{\text {int }}, \beta_{1}\right]$. Thus we only need to show that for any $m$ with $m_{1}<m<m_{0}$ and for all $\beta \in\left[\beta_{0}, \beta_{\text {int }}\right]$,

$$
Q_{m}\left(y_{1: n}\right)+m \beta \geq Q_{m_{0}}\left(y_{1: n}\right)+m_{0} \beta
$$

We show this by contradiction. Firstly assume there exists an $m$ with $m_{1}<m<m_{0}$ and a $\beta \in\left[\beta_{0}, \beta_{\text {int }}\right]$ such that

$$
Q_{m}\left(y_{1: n}\right)+m \beta<Q_{m_{1}}\left(y_{1: n}\right)+m_{0} \beta .
$$

As $m<m_{0}$ and $\beta \leq \beta_{\text {int }}$, this implies

$$
Q_{m}\left(y_{1: n}\right)+m \beta_{i n t}<Q_{m_{0}}\left(y_{1: n}\right)+m_{0} \beta_{i n t},
$$


and by definition of $\beta_{\text {int }}$ we then have

$$
Q_{m}\left(y_{1: n}\right)+m \beta_{\text {int }}<Q_{m_{1}}\left(y_{1: n}\right)+m_{1} \beta_{\text {int }} .
$$

This then contradicts the condition of part (3) of the theorem, namely that a segmentation with $m_{1}$ changepoints is optimal for the penalty $\beta_{\text {int }}$.

Proof of Theorem 3.2 Proof. The proof for part (1) is trivial since we need to run CPD twice, using both $\beta_{0}$ and $\beta_{1}$.

For the proof of part (2) define $N\left(m_{0}, m_{1}\right)$ as the maximum (over data sets) of the number of further runs of CPD needed to find all the optimal segmentations in an interval of $\beta$, given we have run CPD at the lower and upper endpoints of the interval and these have produced segmentations with $m_{0}$ and $m_{1}$ changepoints respectively. As we have run CPD twice, to prove the theorem we need to show that

$$
N\left(m_{0}, m_{1}\right) \leq m_{0}-m_{1}-1
$$

Firstly, if $m_{0}-m_{1}=1$ then $N\left(m_{0}, m_{1}\right)=0$, which satisfies $(0.1)$.

Now we proceed by induction. For an integer $l>1$ assume that if $m_{0}-m_{1}<l$ then (0.1) holds. We need to show that this implies that (0.1) holds for $m_{0}-m_{1}=l$.

In this case our first step is to run PELT at the intersection, $\beta_{\text {int }}$. In the worst case scenario we find that $m\left(\beta_{\text {int }}\right) \neq m_{1}$ (and hence $m\left(\beta_{\text {int }}\right) \neq m_{0}$ as segmentations with $m_{0}$ and $m_{1}$ changepoints have the same penalised cost for penalty value $\left.\beta_{\text {int }}\right)$. We then need to consider the sub-intervals below and above $\beta_{\text {int }}$ separately. Since $m(\beta)$ decreases as $\beta$ increases $m_{0}-m\left(\beta_{\text {int }}\right)<l$ and $m\left(\beta_{\text {int }}\right)-m_{1}<l$. Therefore

$$
\begin{aligned}
N\left(m_{0}, m_{1}\right) & =1+N\left(m_{0}, m\left(\beta_{\text {int }}\right)\right)+N\left(m\left(\beta_{\text {int }}\right), m_{1}\right) \\
& \leq 1+\left[m_{0}-m\left(\beta_{\text {int }}\right)-1\right]+\left[m\left(\beta_{\text {int }}\right)-m_{1}-1\right] \\
& =1+m_{0}-m_{1}-2 \\
& =m_{0}-m_{1}-1 .
\end{aligned}
$$

which satisfies (0.1) as required. 


\section{Further Simulations: Change in mean}

In the main manuscript we looked at a change in mean model with a fixed number of changepoints and compared pDPA to CROPS with FPOP. Here we look at the results when the number of changepoints increases sublinearly and linearly with the number of data points.

(a) Sublinear

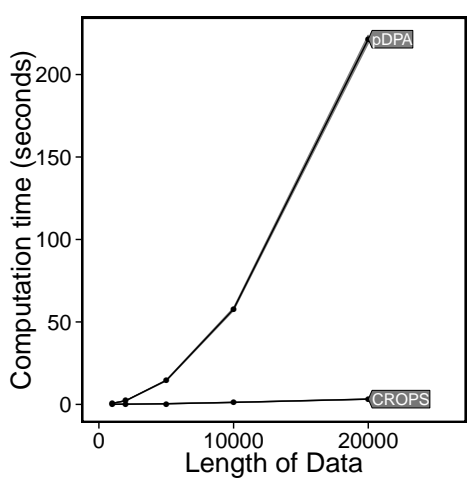

(b) Linear

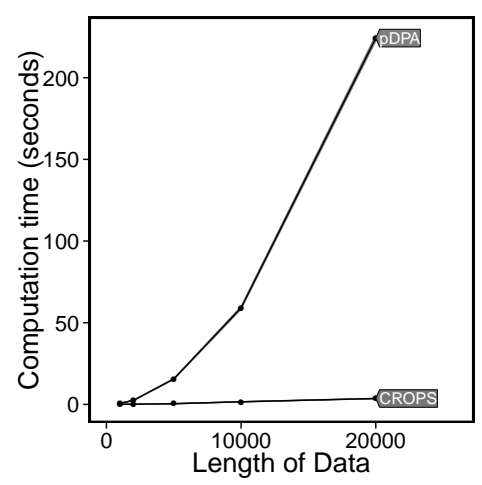

Figure 1: CPU cost for using pDPA and CROPS with FPOP. (a) Sublinear changepoints $(m=\sqrt{n} / 4)$ and (b) linear changepoints $(m=n / 100)$. 


\section{Further Simulations: Change in mean and variance for normal model}

Similarly we revisit the model with a change in mean and variance to compare Segment Neighbourhood with CROPS with PELT and PELT with the recycled calculations for data sets with a sublinear and linear number of changepoints in respect to data length. Figure 2 shows the CPU cost for the 3 methods. We can then use this data and CROPS with PELT to explore penalty choice when we have a different number of changes. These results can be seen in Figure 3.

(a)
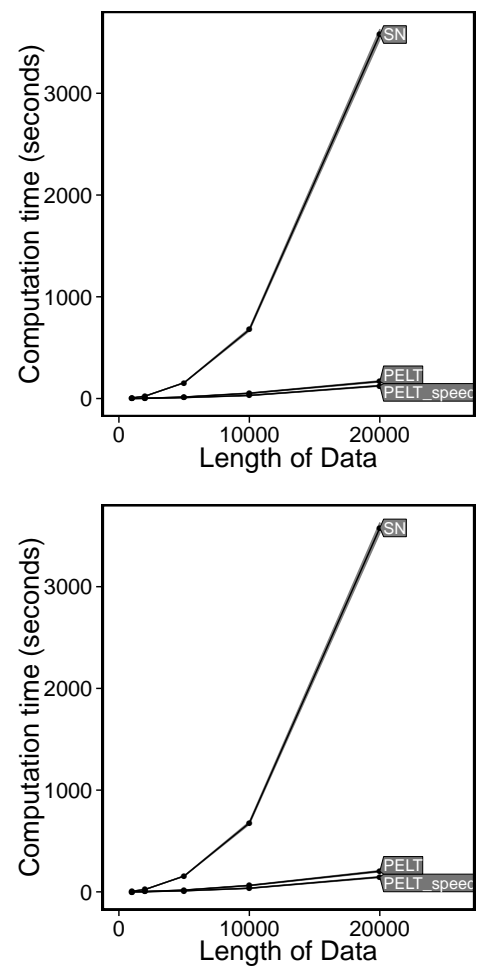

(b)
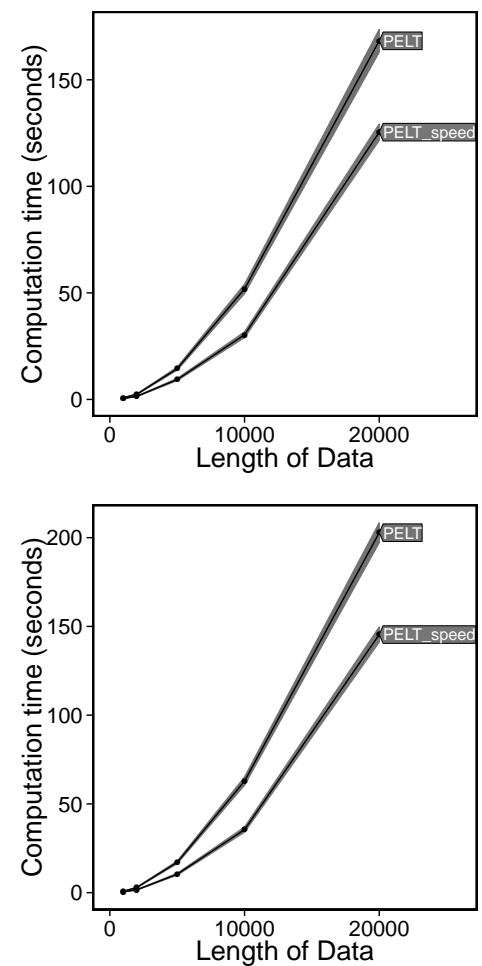

Figure 2: (a) CPU cost for using SN, CROPS with PELT and CROPS with PELT with the speed improvements. (b) A close up of PELT and PELT with the speed improvements. The top row is sublinear changepoints $(m=\sqrt{n} / 4)$ and the bottom row is linear changepoints $(m=n / 100)$. 

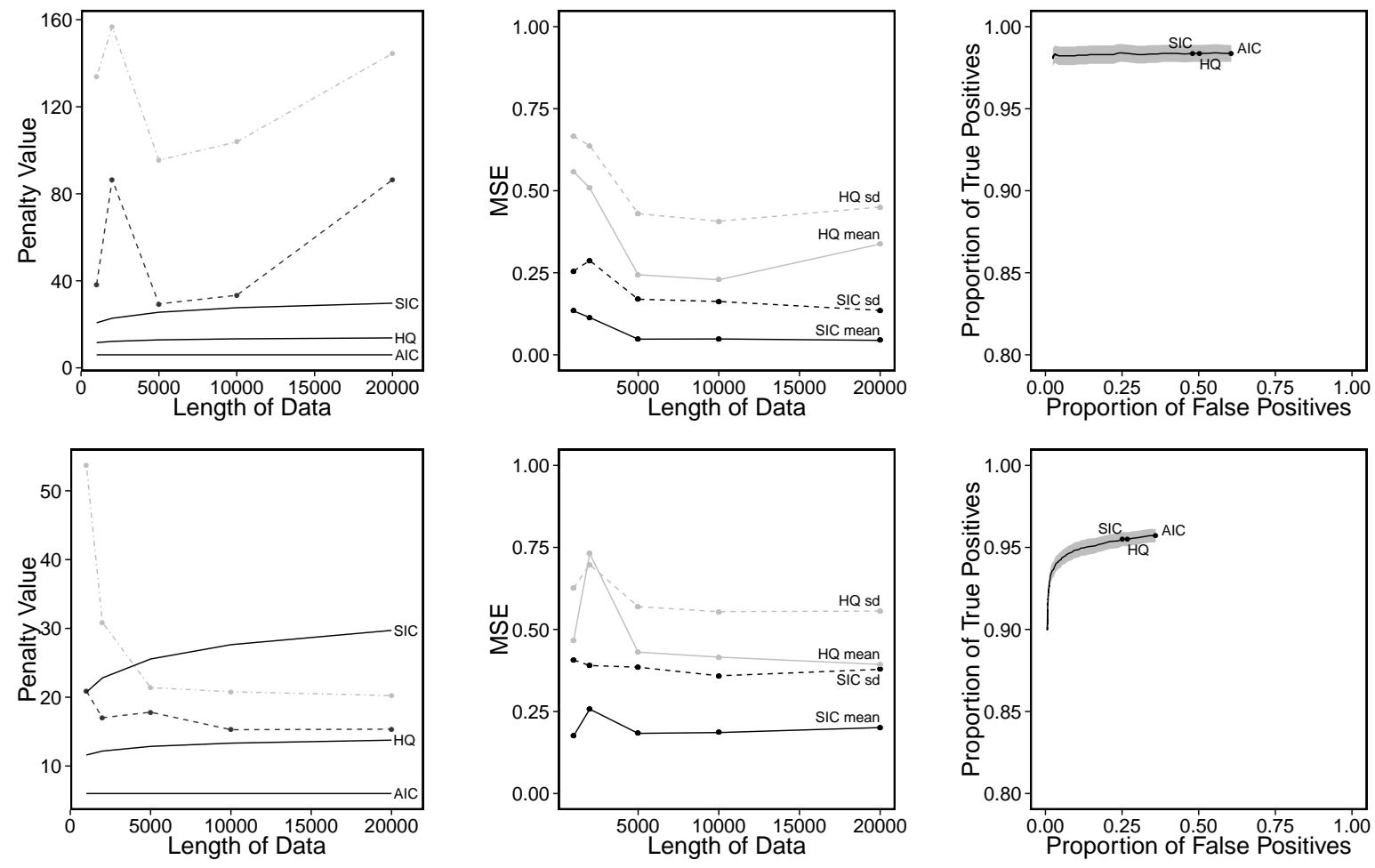

Figure 3: Results for the true model. Left: Average minimum (black, dashed) and maximum (grey, dot-dashed) optimal penalty values in comparison to popular penalty terms in the literature. Solid lines from top to bottom are the SIC, Hannan-Quinn and AIC penalty values. Middle: MSE for the mean (solid) and the standard deviation (dashed) when different penalty terms are used. Right: Proportion of true positives against the proportion of false positives for $n=10,000$. The top row is sublinear changepoints $(m=\sqrt{n} / 4)$ and the bottom row is linear changepoints $(m=n / 100)$. 
Further Simulations: Change in mean and variance for the mis-specified model

We can also look at the situation where we have a mis-specified model with a sublinear and linear number of changes. The range of optimal penalty values and true and false positives found using different common penalty terms are shown in Figure 4
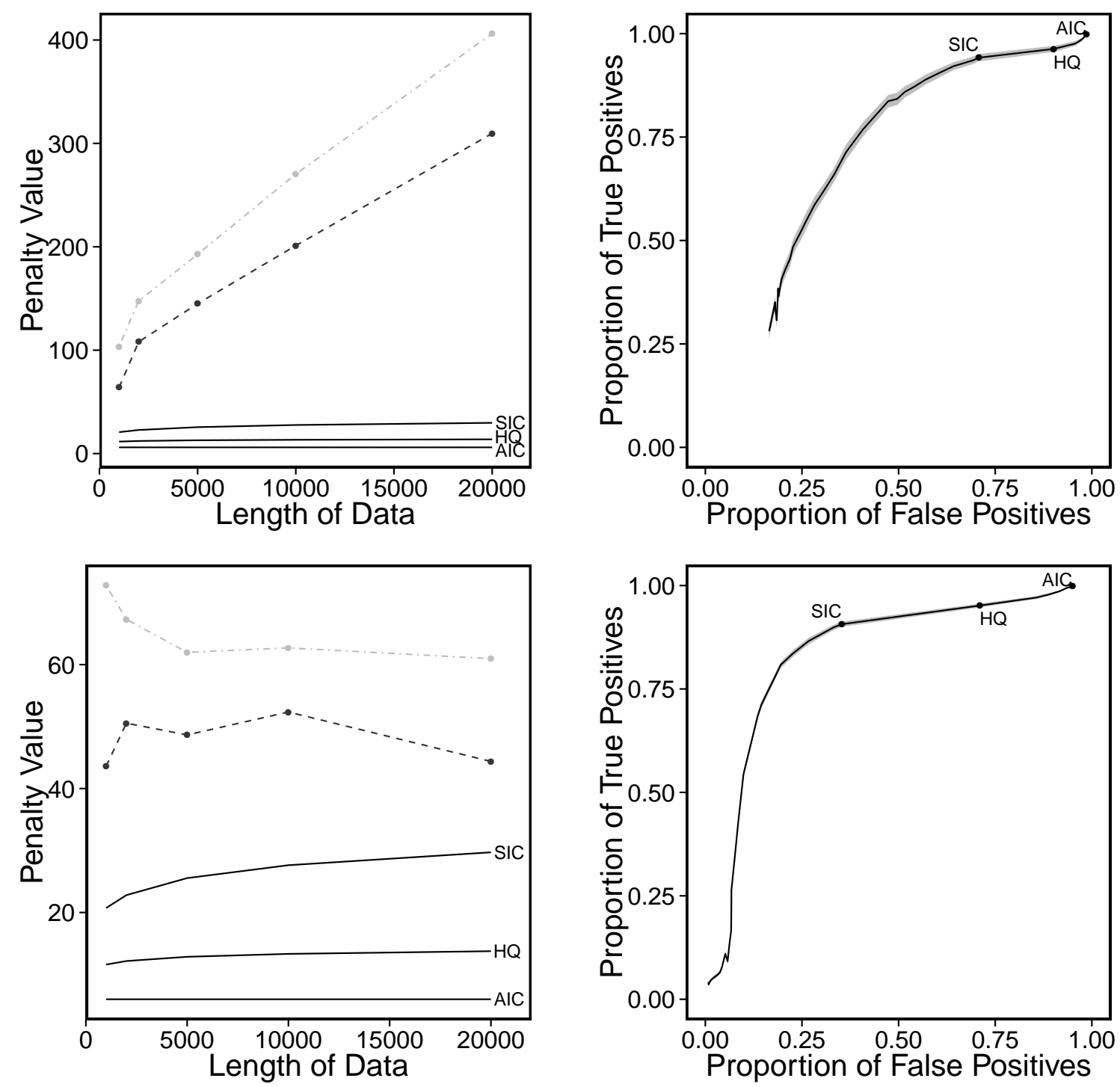

Figure 4: Results for the misspecified model scenario. Left: Average minimum (black, dashed) and maximum (grey, dot-dashed) optimal penalty values in comparison to popular penalty terms in the literature. Right: Proportion of true positives against the proportion of false positives for $n=10,000$. The top row is sublinear changepoints $(m=\sqrt{n} / 4)$ and the bottom row is linear changepoints $(m=n / 100)$. 


\section{Further regions where we have discrepancies in the Hi-C example}

In the real data section of the main manuscript we showed regions of the chromosome where was have detected different segmentations with the different penalty terms. Below are a further 3 regions.

(a)

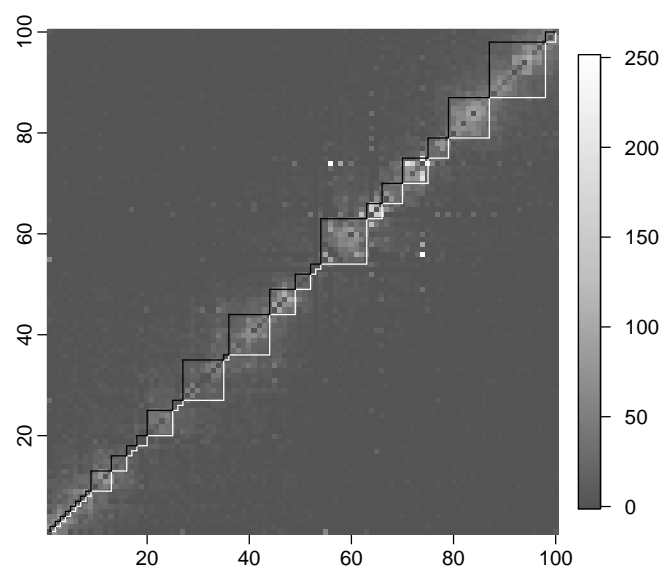

(b)

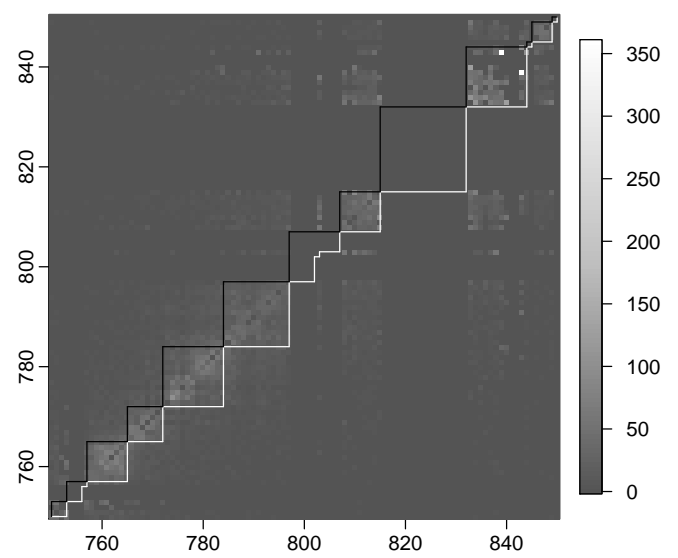

(c)

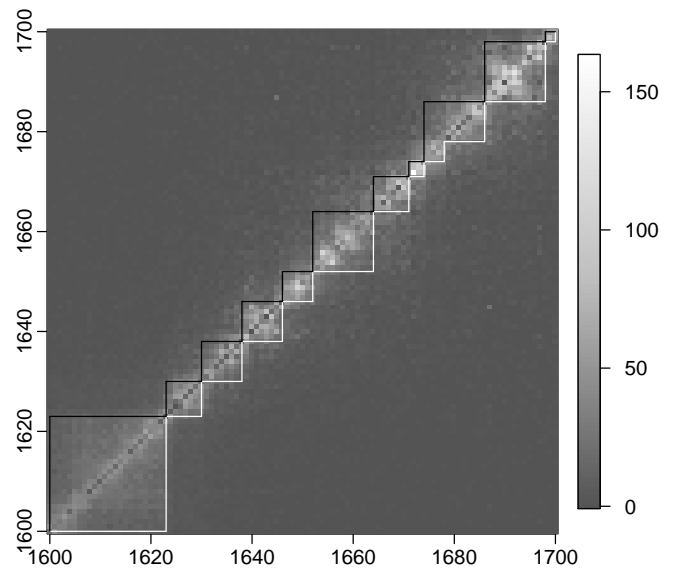

Figure 5: Close up different regions, the black line is the segmentation using our optimal $\beta$ and the grey line is the segmentation using $\beta=0$ and $\beta=$ SIC. 